\title{
Comparative study of thermographic signal reconstruction and partial least squares thermography for the detection and evaluation of subsurface defects
}

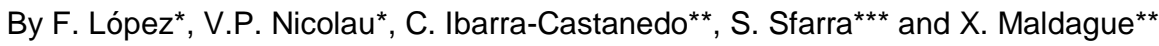

* Dept. of Mechanical Engineering, Universidade Federal de Santa Catarina, 88040-900,Florianópolis, Santa Catarina, Brazil, fernandol@irthermalvision.com

** Electrical and Computing Engineering Dept., Université Laval, Quebec City (Quebec), Canada G1K 7P4, xavier.maldague@gel.ulaval.ca

*** Dept. of Industrial and Information Engineering and Economics, University of L'Aquila, L'Aquila (AQ), Italy I67100, stefano.sfarra@univaq.it

\begin{abstract}
Thermographic signal reconstruction (TSR) and partial least squares thermography (PLST) are techniques conceived for the processing and analysis of pulsed thermography (PT) data. In this work, the capabilities of both techniques are evaluated. A thermal data sequence was obtained from a PT inspection on a carbon fiber reinforced polymer (CFRP) specimen with simulated delaminations (Teflon inserts). The IR sequence was then processed with both TSR and PLST and their results were evaluated in terms of the signal-to-noise ratio at maximum signal contrast for each defect. The discussion focuses on the performance, limitations and advantages of each processing method.
\end{abstract}

\section{Introduction}

Pulsed thermography (PT) has become a widely accepted method for the non-destructive testing and evaluation (NDT\&E) of materials. In this technique, the specimen is submitted to a pulsed thermal excitation while recording the subsequent thermal decay using an infrared (IR) camera. Internal discontinuities, such as delaminations or voids, appear in the form of patterns - or thermal contrasts - on the surface thermal maps. This technique is well known because of its speed and ease of use. Furthermore, it can be applied to several types of materials and structures, such as metallic, polymeric and composite materials. However, the thermal signals acquired with the infrared (IR) camera are often contaminated by different noise sources, the most common being external reflections and surface emissivity variations. In addition, the non-uniform distribution of the irradiation applied during thermal excitation (non-uniform heating), produces abnormal thermal patterns which are often superimposed to the thermal contrasts produced by internal defects. The combination of these physical processes makes more complex the detection and analysis of the thermal images acquired during the PT inspection.

In order to overcome most of the negative effects mentioned above - and consequently to improve the sensitivity to smaller and deeper features - the thermographic signals often require an additional processing step. In general terms, the processing techniques consist in the transformation of the acquired temporal data to a different domain with the purpose of simplifying the data analysis and obtaining a new variable to deal with. This new variable may improve the detection of defects, especially of those which are more sensitive to the effects of lateral heat diffusion and non-uniform heating. Furthermore, quantitative analysis is also possible with this new variable, often providing more accurate results than those obtained with raw data.

Thermographic signal processing is an issue that has been widely investigated. In this regard, several algorithms based on the solution of the 1D equation for heat conduction have been proposed in order to enhance defect visibility [1]. One of the best-known classical PT processing techniques is differential absolute contrast (DAC) [2], which was designed to eliminate the drawback of selecting a sound area when performing classical thermal contrast computations. Pulsed phase thermography (PPT) [3] [4] is one of the techniques which are based on space transformation. Based on the discrete Fourier transform (DFT), in PPT the data is transformed from the time domain to the frequency domain. Phase images constitute the core of PPT analysis since they are less affected by non-uniform heating and surface optical variations than amplitude and raw data.

Thermographic signal reconstruction (TSR) [5] [6] is a processing method that emerged in the early 2000s, providing significant improvements in noise reduction and thus allowing the detection of deeper and smaller defects. The basis of TSR is the use of a low-order polynomial function to fit the temperature evolution cooling profiles obtained from an inspection by PT. One of the most important features of TSR is that it can be used to obtain time-derivative images, which offer important advantages when compared to raw data; their use has been widely reported to increase the detection sensitivity and to reduce the effects of non-uniform heating and the background reflection artifacts.

On the other hand, partial least squares thermography [7] is a recently introduced technique for the analysis and processing of pulsed thermography data. This new method is based on partial least squares regression [8], which decomposes a data array, a thermographic sequence in this case, into a set of orthogonal latent variables. PLST provides a considerable reduction of background noise through the reduction of the non-uniform heating effect from the thermal sequence. 
This works presents a comparative analysis between TSR and PLST. The discussion is focused on the application, capabilities and limitations of both processing techniques. To this aim, a thermal data sequence was obtained from a PT inspection on a carbon fiber reinforced polymer (CFRP) specimen with simulated delaminations (Teflon inserts) and then processed by both TSR and PLST. Results are evaluated in terms of signal-to-noise ratio (SNR) at maximum signal contrast for each defect. Numerical simulation results are also used to widen the discussion and analysis to the fundamentals concepts of heat transfer in PT. The influence of inspection parameters - such as acquisition frequency and truncation window - on the performance of both techniques are also discussed.

\section{Experimental approach}

In this work the experimental approach consisted in the use of two photographic flash lamps (Balcar FX60, giving $6.4 \mathrm{~kJ}$ for $15 \mathrm{~ms}$ each, $2 \mathrm{~ms}$ at full width half maximum) to produce a high-power short pulse used to stimulate the specimen surface. Both flashes were located $50 \mathrm{~cm}$ from the specimen surface. Acquisition of thermal images was carried out by an FPA infrared camera (Santa Barbara FPA SBF125, 3 to $5 \mu \mathrm{m}$, with a $320 \times 256$ pixel array) using a synchronization unit, which accurately controls the time between the launch of the thermal pulse and the recording with the IR camera. Thermal images were stored in a PC for processing and analysis. The experimental system is depicted figure 1.

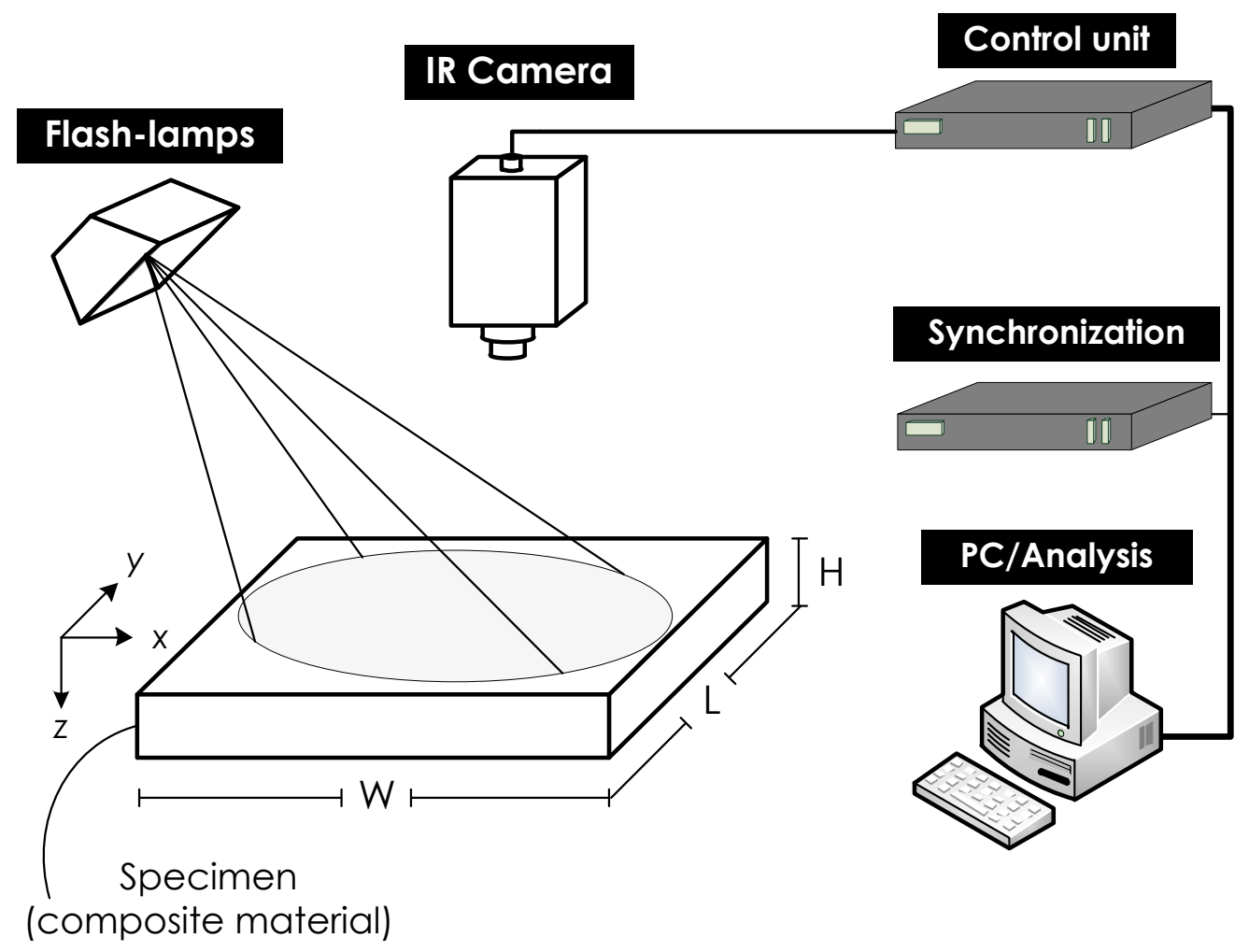

Fig. 1. Experimental setup for pulsed thermography inspection.

In order to exploit all the resources of the IR camera, the specimen was inspected using a fixed acquisition frequency of $157 \mathrm{~Hz}$, which is the maximum full-frame (320x256 pixel array) rate achieved by this camera. Furthermore, a total of 1000 frames - maximum storage capacity of the IR camera - were collected during the inspection and then processed using the techniques under discussion in this work. The academic specimen used in this investigation is a carbon fiber reinforced polymer (CFRP006). The specimen consists of a 10-plies fiber reinforced polymer with 25 Teflon square insertions located at different depths $(0.2<z<1.0 \mathrm{~mm})$ and having different lateral sizes $(3 \leq D \leq 15 \mathrm{~mm})$. The variable adopted in this work to analyze and discuss the results is the aspect ratio $(D / z)$, which ranges from 3 to 75 . 


\section{Theoretical analysis}

As already mentioned the inspection by PT is highly affected by optical reflections, emissivity variations, nonuniform heating and lateral heat diffusion. Some of these limitations are consequence of the nature of the IR measurement, while others are produced by the experimental setup and the thermal process within the specimen. This section provides a theoretical background of two of the major drawbacks in PT: non-uniform heating and lateral heat diffusion.

\subsection{Non-uniform heating}

Non-uniform heating is one of the major - if not the most important - source of uncertainty in pulsed thermography. Even when a flat surface is inspected, several factors as heating source locations (related to the IR radiation sources), equipment aging, external heating or cooling sources (both related to ambient conditions), uneven optical properties of the surface, etc., will produce irregular patterns of heating. These irregular patterns often superimpose to the contrasts produced by internal defects, consequently affecting both the minimum size of the defect that can be detected and the limit of detection (maximum depth that can be reached). Moreover, given that most of the quantitative methods are based on temperature differences, non-uniform heating could affect the accuracy in the prediction results.

Figure 2 exemplifies how the visibility of the defects (in terms of thermal contrast) is reduced due to the presence of non-uniform heating, especially at the center of the specimen. Two sets of simulated thermograms are shown: on the top the thermograms were obtained when the distribution of the applied irradiation is uniform (which physically is almost impossible to obtain), while a non-uniform irradiation distribution was added to the thermograms at the bottom line of this figure. The numerical results were obtained through the solution of the 3D heat conduction equation for anisotropic media, considering the CFRP006 specimen. All aspects concerning to the thermal model and numerical solution approach are detailed in references [9] and [10].

As shown in figure 2, the irregular pattern produced by the non-uniform heating is present since the beginning of the acquisition (at $0.0064 \mathrm{~s}$ which corresponds to the first frame), affecting primary the defects that are located at the center of the specimen. At $0.0318 \mathrm{~s}$ (the fifth frame), the defects located at $0.4 \mathrm{~mm}$ can be detected when the applied surface irradiation is uniform; however, the thermogram obtained when the applied heating is non-uniform does not reveal the presence of these defects. As times elapses, the negative effects of non-uniform heating are more evident. This can be observed at $0.191 \mathrm{~s}$ (corresponding to the thirtieth frame of the acquisition), where it can be noticed how the thermal contrast of all the defects are reduced compared when the surface irradiation is uniform.

As already shown, along with the reduction of the thermal contrast (consequently the visibility of the defects), non-uniform heating can also delay the appearance of the defects (the time at which the defect becomes first visible). The last mentioned affects the deeper defects and combined with the lateral heat diffusion, produces the blurring, which is the degradation of the defect signal.
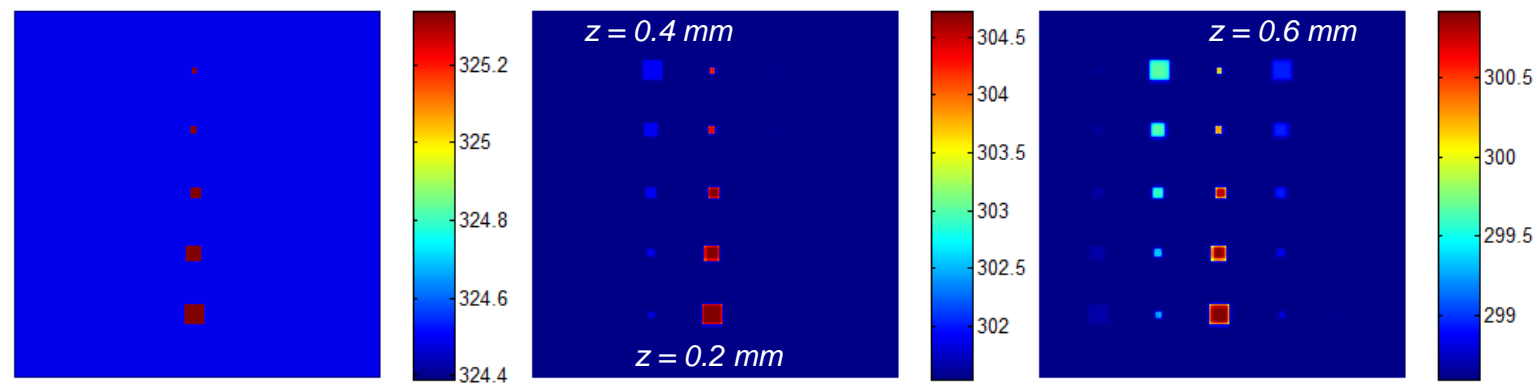

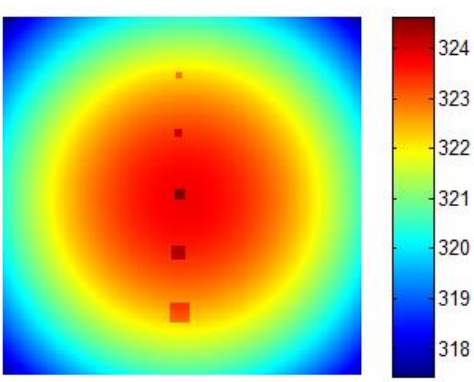

$\mathrm{t}=0.0064 \mathrm{~s}$

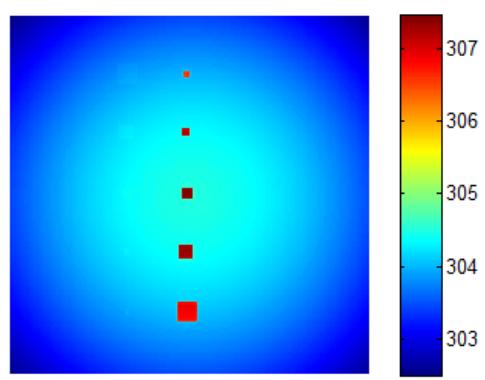

$t=0.0318 s$

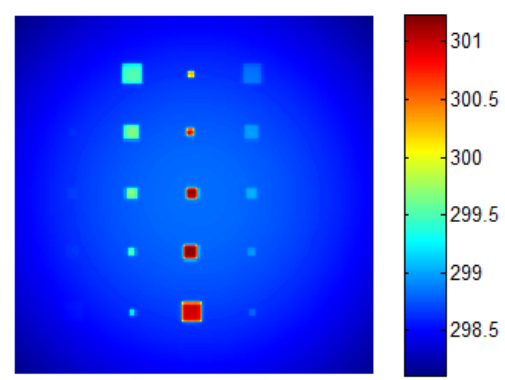

$t=0.191 s$

Fig. 2. Comparison between simulated thermograms obtained by numerical simulation considering non-uniform (top) and uniform (bottom) distribution of irradiation. Results obtained at different times of the cooling process regime. 


\subsection{Lateral heat diffusion}

Lateral heat diffusion is an unavoidable problem in the PT inspection. It is inherent to the heat diffusion process that takes place within the specimen and is dominated basically by the thermal properties of the material and characteristics associated to the defects (depth and lateral size). Blurring is the signal degradation and represents the major negative effect affecting mostly the deepest defects. It becomes significant when the heat conduction in the lateral direction becomes predominant in the diffusion process. Considering the 3D heat diffusion problem in anisotropic media, the Fourier number for each direction $(x, y$ and $z$ ) can be written as follows:

$$
\begin{aligned}
& F o_{x x}=\frac{\alpha_{x x} t}{D^{2}} \\
& F o_{y y}=\frac{\alpha_{y y} t}{D^{2}} \\
& F o_{z z}=\frac{\alpha_{z z} t}{z^{2}}
\end{aligned}
$$

In Eqs. (1) to (3), $\alpha_{x x}, \alpha_{y y}$, and $\alpha_{z z}$ are respectively the thermal diffusivity in the $x-, y-$ and $z-$ directions. Furthermore, $D$ and $z$ are the lateral size and depth of the defects, respectively, whilst $t$ is the time. The degradation of signal - or blurring - takes place when heat diffusion in the $x$ - and $y$-directions become predominant, or,

$$
F o_{x x}, F o_{y y} \gg F o_{z z}
$$

By analysing each of the terms of Eqs. (1) to (3), it can be observed that deeper defects (or those with smaller aspect ratio) are more likely to suffer from blurring. Furthermore, the thermal diffusivity of the material plays an important role in the particular case of an anisotropic material such as CFRP, since for the CFRP006 specimen $\alpha_{x x}, \alpha_{y y} \gg \alpha_{z z}$.

\section{Thermographic signal processing}

As already reviewed, PT - in its basic form - is generally limited to qualitative applications on large, near-surface defects. For this reason, signal processing is a necessary step in order to overcome the effects of non-uniform heating and lateral heat diffusion. This section provides the fundamentals concepts of TSR and PLST, which are subject of investigation in this work.

\subsection{Thermographic signal reconstruction - TSR}

TSR is a popular and an attractive processing method specially conceived to be used with PT data [5] [6]. This technique brings important improvements and advantages over PT raw data, the most significant being the simplicity and accuracy of quantitative measurements, increase of temporal and spatial resolution, reduction of high frequency noise and the ability to produce time derivative images without generating additional noise. The regression proposed by Shepard [5] consisted on the use an $m$-degree polynomial function to reconstruct - or to fit - the logarithmic time dependence of thermographic data. This polynomial function can be written as:

$$
\ln \left(T-T_{o}\right)=a_{o}+a_{1} \ln (t)+a_{2}[\ln (t)]^{2}+\cdots+a_{m}[\ln (t)]^{m}
$$

As can be observed in Eq. (5), one of the main steps in the regression process in TSR is the selection of the appropriate number of coefficients $m$ to fit the thermographic data. For isotropic materials, a good correspondence between acquired data and fitted values can be achieved by setting $m$ to 4 or 5 [11], given the number of inflections points of the typical PT thermal profiles. Nevertheless, when working with anisotropic materials such as those considered in this work (carbon fiber reinforced polymers) the number of coefficients should be carefully selected in order to avoid higher residuals between fitted and experimental data, especially in the transient regions where the effects of lateral heat diffusion are not still predominant. The parameter used in this work to measure the quality of the TSR fitting model is the root mean square error (RMSE). Figure 3 shows the residuals of the fitted model and the RMSE as a function of the numbers of coefficients. It can be shown that the TSR regression with only three coefficients has large residuals at the beginning of the cooling process (see figure 3a). With six components the residuals and the RMSE are reduced but still are present higher oscillations, especially at times where the thermal regime can be considered 1D (see for instance, figure $3 b$ ). Based on the previous analysis, the optimum number of coefficients used to fit the thermographic data was set to $m=8$. This number ensures a good correspondence between fitted and experimental data and also avoids the ringing (higher-order oscillation). 


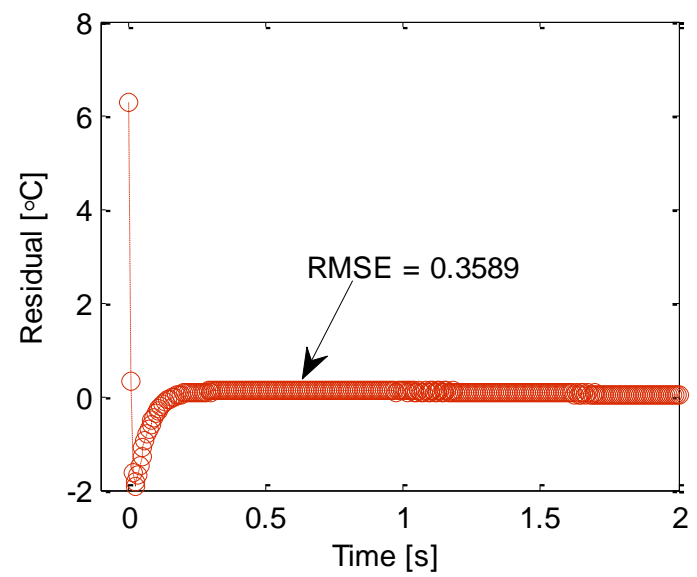

(a)

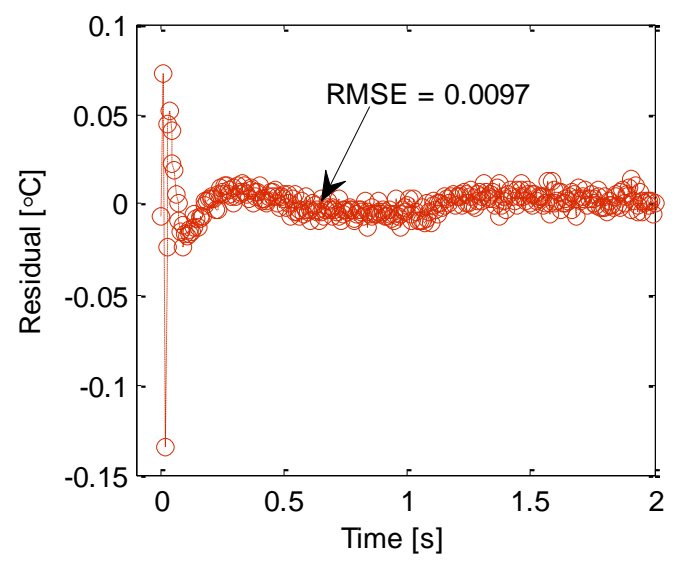

(c)

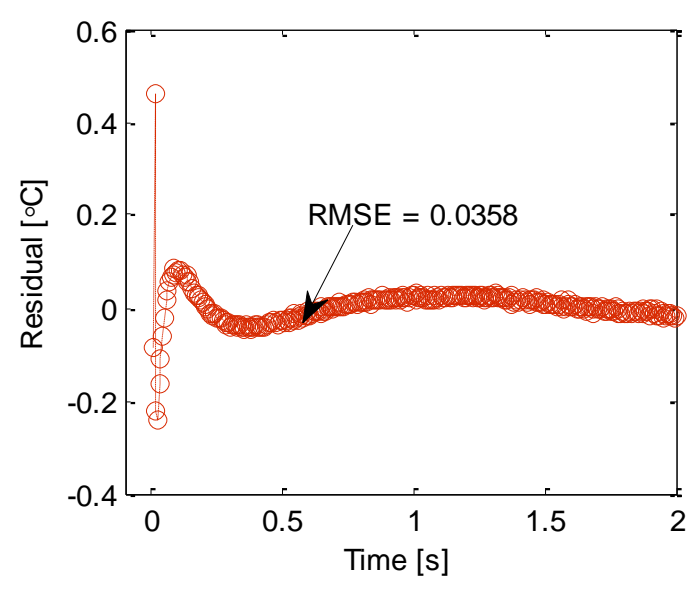

(b)

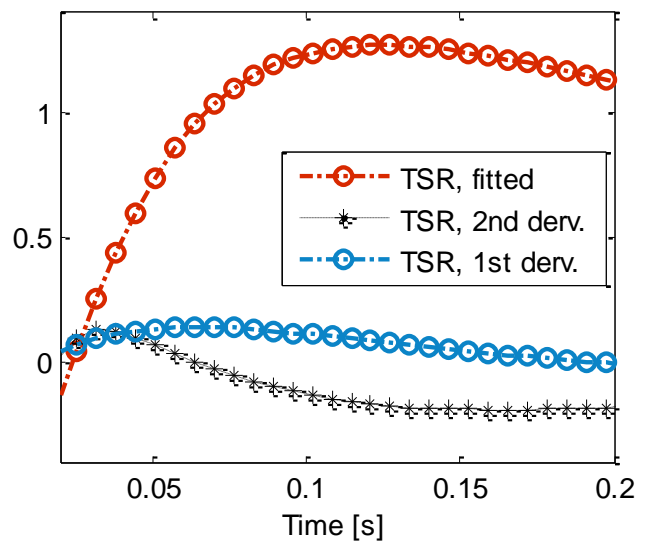

(d)

Fig. 3. Residuals along time and root mean square errors as a function of the numbers of coefficients in the regression eq. (5), considering (a) $m=3$, (b) $m=6$, (c) $m=8$. (d) Contrast curves for fitted, first and second derivative considering a defect with $D / z=10 / 0.2$

As mentioned earlier, one of the main attractions of TSR is its ability to produce time derivative images, which provide a considerably reduction in background noise [15]. With time derivatives images it is also possible to reduce the onset time and the time of maximum signal contrast of the defects, thus reducing the blurring effect. This is exemplified in figure $3 \mathrm{~d}$, where are plotted the contrast curves for the fitted, first and second time derivatives of the defect with $\mathrm{D} / \mathrm{z}=$ $10 / 0.2$ As observed in figure 4, which presents a series of thermograms from a PT experiment, about $96 \%$ of the defects were detected with TSR first-time derivative images. It is worth mentioning that the shape of the defects is still preserved and the most important, the performance of this technique can be improved when optimizing the acquisition parameters.

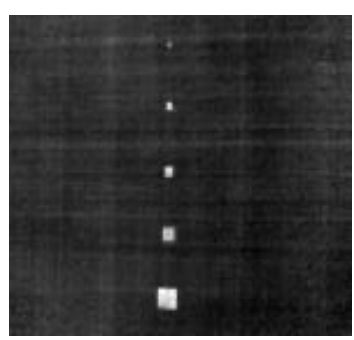

$0.203 \mathrm{~s}$

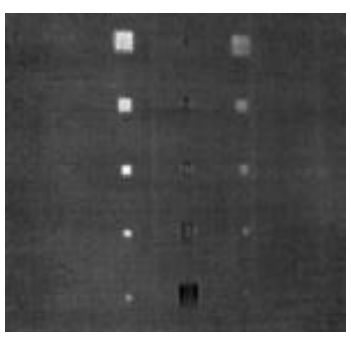

$0.254 \mathrm{~s}$

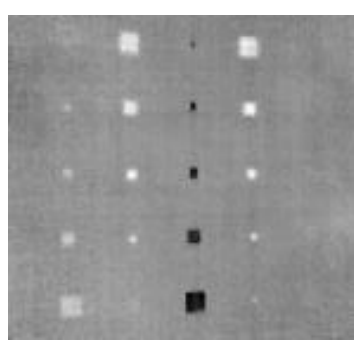

$0.350 \mathrm{~s}$

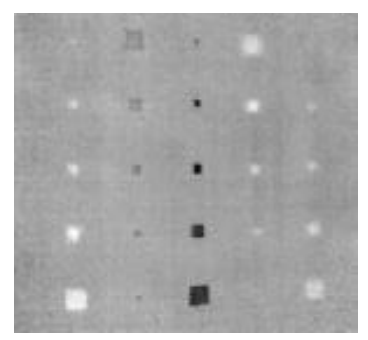

$0.668 \mathrm{~s}$

Fig. 4. First-time derivative images for different times obtained after the implementation of TSR. 


\subsection{Partial least squares thermography - PLST}

In spite of the great improvement in the quality of the images obtained with the processing techniques based on the 1D solution of the heat conduction equation (for instance, DAC and TSR), their application is subjected to certain criteria, which include: defect depth and lateral size, thermophysical properties of the material and the duration of the transient regime. This is due to the fact that these techniques consider that the transient thermal behavior is 1D, which implies that the heat diffusion process in the transversal direction is the most predominant. This condition is not always satisfied and usually deeper defects are more affected by 3D heat diffusion [see the terms of eqs. (1) to (3)]. These concerns motivated the review of an alternative method that could allow the reconstruction of the thermographic signatures while maintaining physical consistency.

Based on a statistical correlation method, partial least squares thermography (PLST) has been proposed as a technique for the treatment of thermographic images [7]. PLST computes loading $P$ and score $T$ vectors that are correlated to the predicted block $Y$ (as in maximum redundancy analysis), while describing a large amount of the variation in the predictor matrix $X$ (as in principal component regression). The matrix $X$ corresponds to the surface temperature matrix obtained during the PT inspection, while $Y$ is defined by the observation time during which the thermal images were captured. The PLS model is achieved by decomposing $X$ and $Y$ into a combination of loadings $P$ and $Q$ (formed by orthogonal vectors), scores $T$ and $U$ (the projections of the loading vectors associated with the singular values) and residuals $E$ and $F$ [12] [13]. Mathematically, the PLS model is expressed as:

$$
\begin{aligned}
& X=T P^{\prime}+E \\
& Y=U Q^{\prime}+F
\end{aligned}
$$

The bilinear decomposition of eqs. (6) and (7) is carried out using the NIPALS algorithm [14], which computes scores, weights, loadings and inner-coefficients sequentially. In order to perform the decomposition of the thermal sequence matrix (which in a fact is a regression) it is necessary to select the appropriate number of PLS components. To this aim two parameters must be taken into account: the RMSE and the percentage variance explained in $X$ matrix.

Figure 5 shows the computation results of both variables of the PT thermal sequence. It can be observed that although the minimum RMSE is reached with 10 components, only 6 components are sufficient to describe all of the variance in the predictor matrix $X$. Increasing to more than 6 PLS components will introduce higher-order oscillations into the new data sequence.

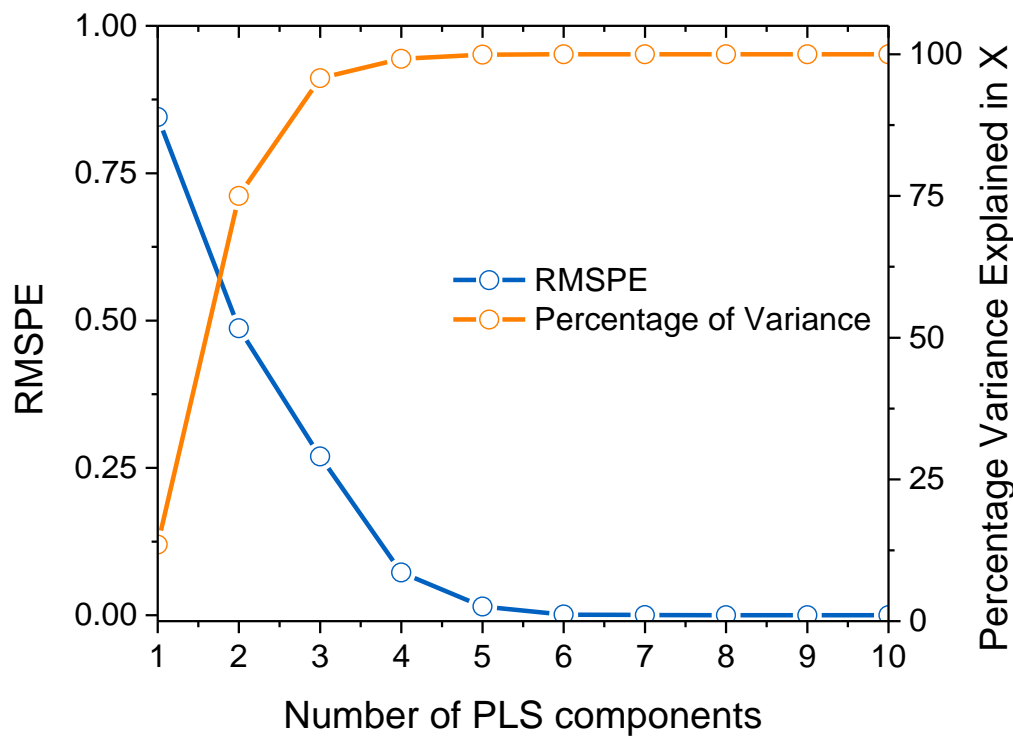

Fig. 5. RMSE and percentage variance explained in X matrix as a function of PLS components.

One of the main attractions of PLST is the separation of physical effects. This is because of the orthogonality between each latent variable obtained from the decomposition of the thermal sequence. This can be exemplified in figure 6 where the six X loadings are shown, each one containing the coefficients that define a linear combination of PLS components that approximate the original thermal sequence. It can be noted from figure $6 a$ that the first PLS loading conserves a Gaussian-shape distribution, with higher values for the coefficients at the center of the images. This behaviour indicates that the effect of non-uniform heating is retained in this PLS component. 


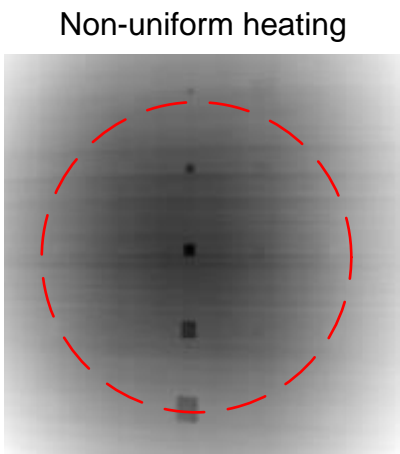

(a)

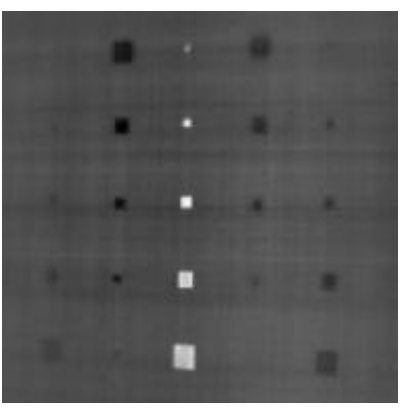

(d) $\mathrm{z}=0.2 \mathrm{~mm}$

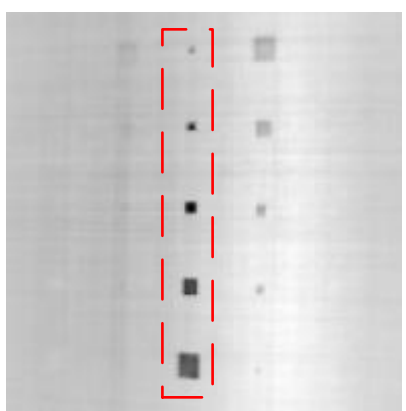

(b)

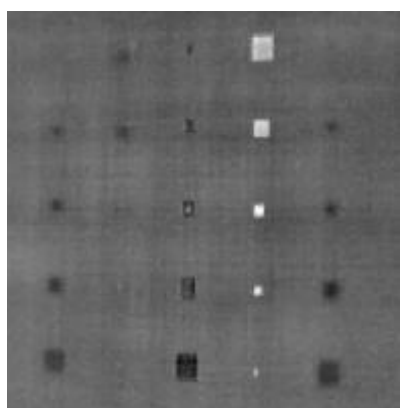

(e) $\mathrm{z}=0.4 \mathrm{~mm}$

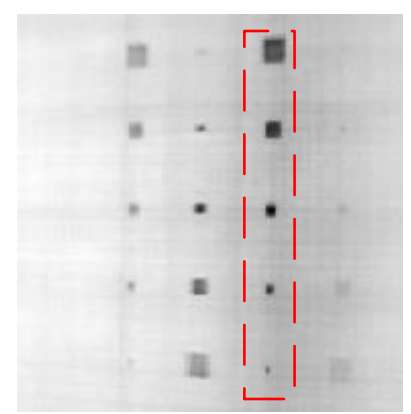

(c)

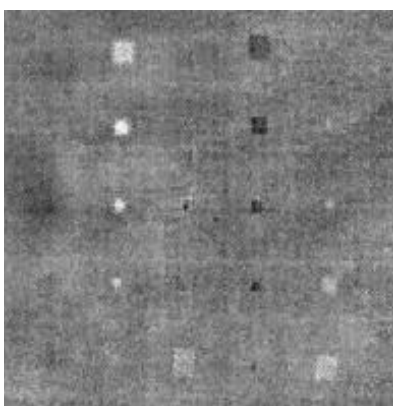

(f)

Fig. 6. PLS loadings obtained after the implementation of PLSR to the experimental pulsed thermography data on the CFRP006 specimen.

The second PLS loading (see figure $6 \mathrm{~b}$ ) shows that defects located at $0.2 \mathrm{~mm}$ can be clearly detected; similarly, most of the defects with $\mathrm{z}=0.4 \mathrm{~mm}$ can be detected in the same loading but with less contrast. Deeper defects can be observed in the third, fourth and fifth PLS loadings (respectively figure $6 c, d$ and e), which is due to the fact that the variation in the temperature during the cooling process becomes lower as the depth increases (in agreement with heat conduction theory). From the analysis of the loadings and the behaviour of each component along the time, it is possible to identify the difference sources of variability. Figure 7 shows the results of this analysis, where it was possible to determine that the first PLS component retains the highest variability (associated to the non-uniform heating), whilst the variability of the second PLS is associated to the heat diffusion process.

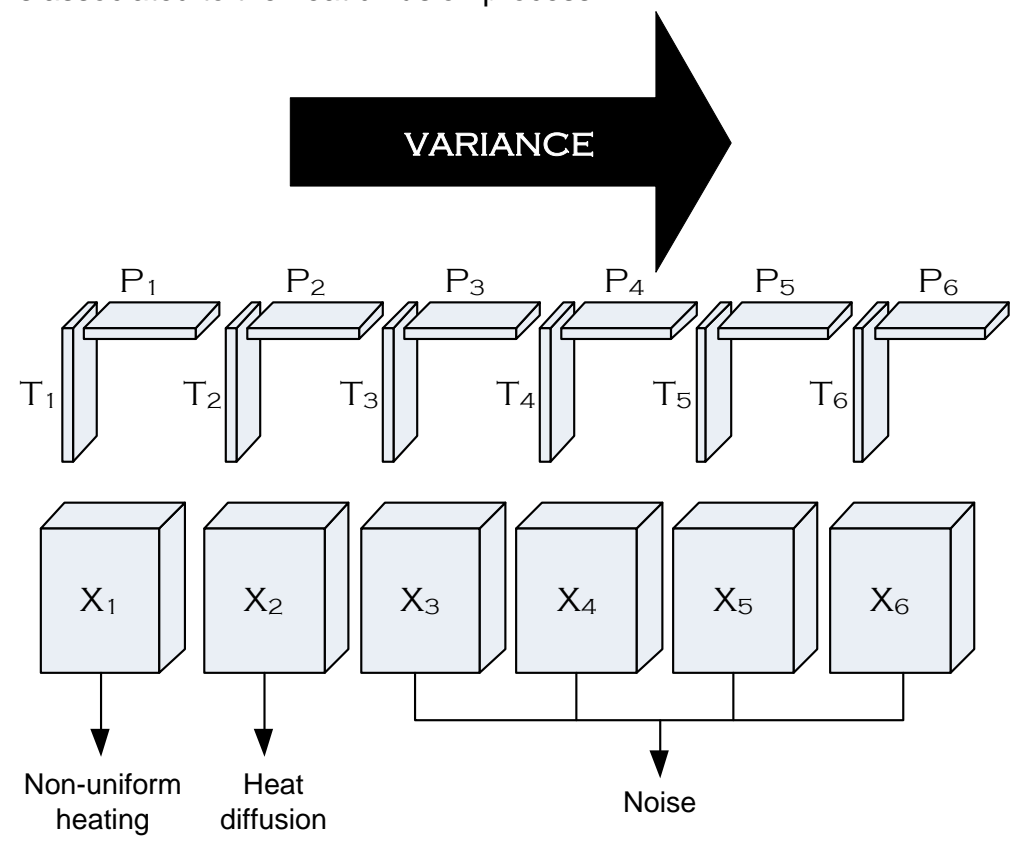

Fig. 7. Difference sources of variability found after de decomposition of $X$ into latent variables. 
Based on the previous analysis, the $1^{\text {st }}$ PLS component, which retains the highest variance produced by nonuniform heating, is suppressed from the synthetic data. In other words, non-uniform heating effects from the original thermal data are diminished. The results of this operation are shown in figure 8 where it can be noted that after the suppression of the $1^{\text {st }}$ PLS component for the synthetic data, a considerable enhancement in the visibility of the defects is achieved. From the first frame of the thermal sequence (at $0.0064 \mathrm{~s}$ ) the defects located at $0.2 \mathrm{~mm}$ are already visible in the enhanced thermal sequence. At 0.0446 seconds (second frame), defects located at 0.4 and $0.6 \mathrm{~mm}$ depth are visible, even those with the smallest lateral size. Moreover, it is important to note that the Gaussian distribution of temperature produced by the non-uniform excitation was eliminated from the new sequence, allowing defects located close to the edges of the specimen to be easily detected. At $0.9936 s$ almost $96 \%$ of the defects are clearly visible, which represents a significant improvement in the ability of PLSR to detect defects at earlier times when compared with $32 \%$ of detection when the non-uniform effect is present in the data sequence.
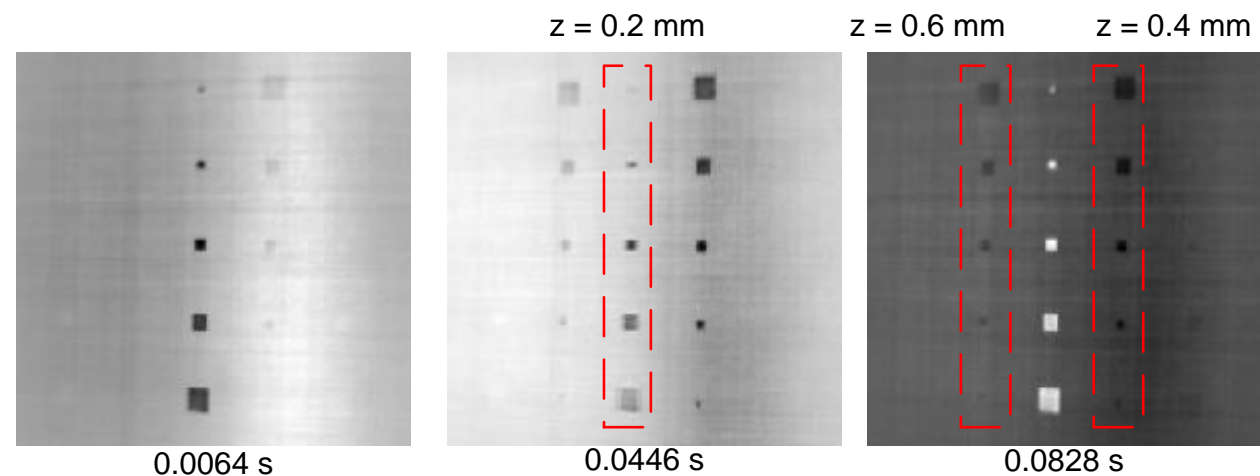

$0.0828 \mathrm{~s}$

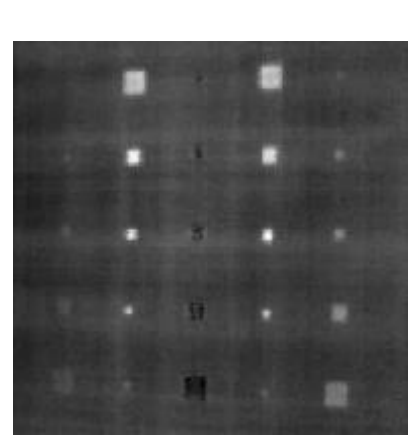

$\mathrm{z}=1.0 \mathrm{~mm} \quad \mathrm{z}=0.8 \mathrm{~mm}$

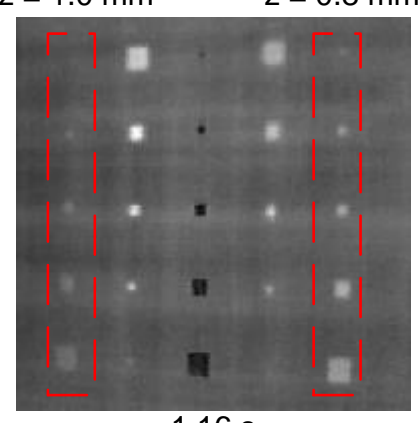

$1.16 \mathrm{~s}$

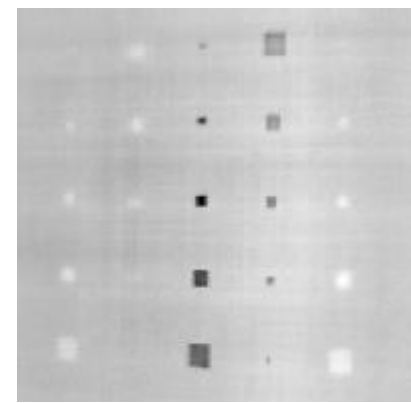

$1.75 \mathrm{~s}$

Fig. 8. Enhanced thermal sequence after suppression of the non-uniform heating effects in the PT data of the CFRP006 specimen.

\section{Comparative analysis}

Thermographic signal reconstruction is a well-positioned technique for the processing of thermographic images. This technique has a proven-track record to be one of the most robust and easy to implement even in badly sampled data. For this reason TSR has been the selected technique to perform a comparison in order to measure the capabilities of PLST. The variable adopted in this work to evaluate the performance of both techniques is the signal-to-noise ratio at maximum signal contrast. The methodology implemented to perform the computation of $S N R$ at $C_{\text {max }}$ is described in reference [1].

The results of the $S N R$ at $C_{\max }$ for raw, TSR and PLST data are shown in figure 9. Firstly, it is important to note that the SNR of defects with aspect ratio of 3, 7 and 10 in raw data is zero. This is due to the fact that, in the case of defects with $D / z=3$ and 7 , there was not enough contrast to detect them, and, in the case of the defect with $D / z=$ 10 , the noise level was higher than the signal amplitude. Another important fact that should be mentioned is that the SNR obtained from raw data is above 20 decibels for most of the defects, which is considered to the detection threshold. 


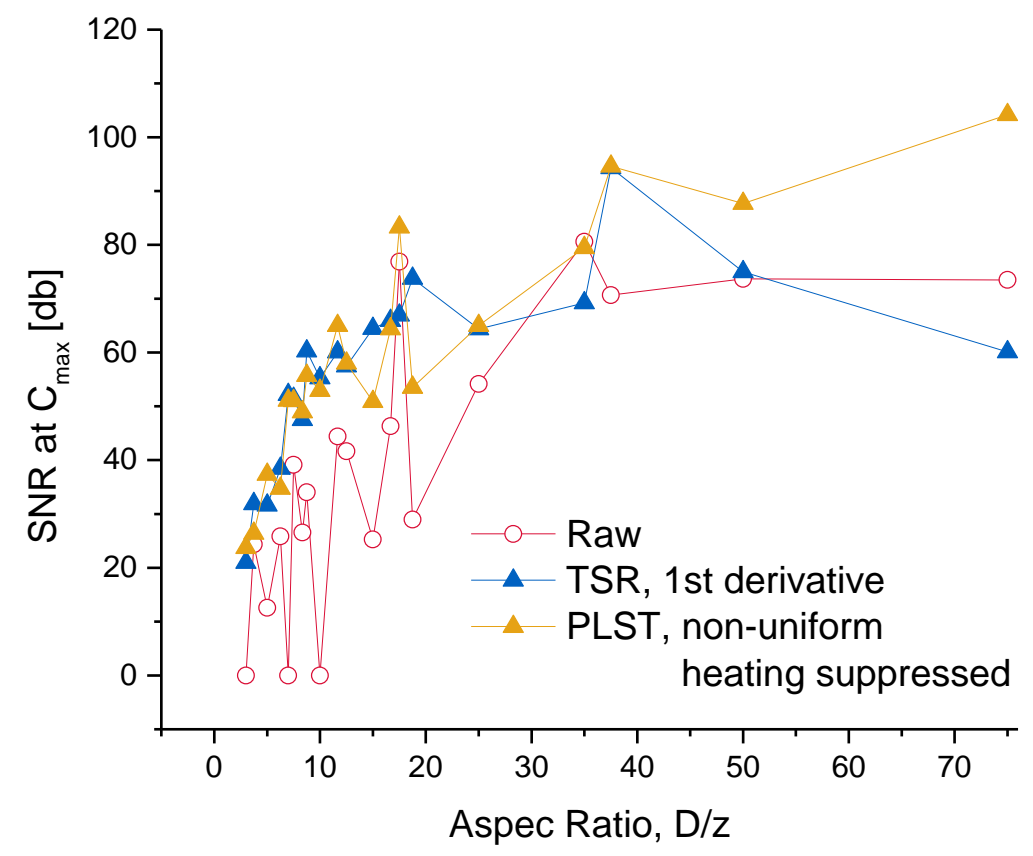

Fig. 9. Results of the signal-to-noise ratio at maximum signal contrast as a function of defect aspect ratio.

The real usefulness of processing data becomes more evident for defects having the smallest aspects ratios. In those cases, an appreciable enhancement of the SNRs at maximum signal contrast were achieved with the treatment of the thermal images using both, TSR first-time derivative and PLST with non-uniform heating supressed, especially for those defects with aspect ratio below 10 (more challenging defects to be detected). With PLST an increase of the SNR in $96 \%$ of the defects was achieved, whilst with TSR $1^{\text {st }}$ derivative an increase in SNR was observed in $94 \%$ of the defects. In general terms, both techniques present similar SNR values for defects with smaller aspect ratio. Only for defects with $D / z=50$ and 75 , PLST obtained SNR values considerable higher than TSR. However, this fact does not compromise the performance of TSR since these defects are easily detectable with raw data.

It is important to recognise that in TSR, the acquisition parameters may contribute to obtain better SNR values (since TSR suffer the potential consequences of undersampling). As for PLST, the effects of the sampling frequency and truncation window on the SNR still need to be investigated.

Since TSR is derived from the 1D heat conduction equation (which assumes that the thermal process is dominated by the heat diffusion in the transversal direction), the regression fitting can be affected when considering longer times (affecting deeper defects), when the lateral heat diffusion becomes predominant [see eqs. (1) to (3)]. In the particular case of this work, the SNR results obtained with TSR $1^{\text {st }}$ time derivative showed a good performance when considering depths between $0.2 \mathrm{~mm}$ and $1.0 \mathrm{~mm}$. With regards to PLST, since it is based on the variability of temperature profiles (rather than physical-based concepts) in theory it can be applied to those cases which require longer observation times. This issue has to be further investigated as well.

\section{Conclusions}

In this work, the fundamentals concepts of thermographic signal reconstruction and partial least squares thermography - a recently introduced technique for the processing and analysis of thermographic data - have been reviewed. Along with details concerning to the experimental approach, a section was dedicated to discussing the major drawbacks in pulsed thermography, making use of heat transfer theory.

The implementation of these techniques was carried out on raw data obtained from a pulsed thermography inspection of a carbon fibers reinforced polymer and then the performance of both techniques were evaluated in terms of the signal-to-noise ratio at maximum signal contrast. The results showed that a considerable increase on the SNR values can be obtained for deeper and smaller defects after the implementation of both techniques on the PT raw data. Furthermore, the obtained SNRs for defects with smaller aspect ratio were similar for both techniques; only in two cases the SNR of PLST was considerable higher than the obtained with TSR. However, this result should not be considered as a demonstration of an improved performance of PLST over TSR since these defects in particular are easily detectable even with raw data. A more profound work is required in order to investigate in more details the impact of the acquisition parameters (frame rate, temporal window) over the performance (SNR) of both techniques. 
Optimizing this, an interesting perspective of PLST could be the analysis of thermograms acquired into the cultural heritage field. In particular, would be interesting to compare IRT results coming from techniques previously discussed and linked to wooden and frescoes artworks [15].

\section{Acknowledgements}

The authors would like to thanks the financial support provided by the CNPq (Science without Borders Program) of the Brazilian government as well to the Foreign Affairs and International Trade Canada (Emerging Leaders of Americas Program). The authors are also grateful with the Canadian Research Chair Multipolar Infrared Vision (MiViM) and Natural Science and Research Council of Canada (NSERC) for providing the support and all the laboratory equipment to carry out the experiments.

\section{REFERENCES}

[1] F. López, C. Ibarra-Castanedo, M. X. and V. Nicolau, "Pulsed thermography signal processing techniques based on the 1D solution of the heat equation applied to the inspection of laminated composites," Materials Evaluation, pp. 91-102, 2014.

[2] M. Pilla, M. Klein, X. Maldague and A. Salerno, "New Absolute Contrast for Pulsed Thermography," in Proc. Quantitative Infrared Thermography Conference, Dubrovnik, Croatia, 2002.

[3] X. Maldague and S. Marinetti, "Pulse Phase Infrared Thermography," J. Appl. Phys., 79(5):2694-2698, 1996.

[4] Ibarra-Castanedo C. and Maldague X. "Pulsed Phase Thermography Reviewed," QIRT J., 1(1):47-70, 2004.

[5] S. M. Shepard, "Advances in pulsed thermography," in The International Society of Optical Engineering, Thermosense XXVIII, Orlando, FL, 2001.

[6] S. M. Shepard, "Flash Thermography of Aerospace Composites," in IV Conferencia Panamericana de END, Buenos Aires, 2007.

[7] F. López, V. Nicolau, M. X. and C. Ibarra-Castanedo, "Multivariate Signal Processing Technique by PartialLeast Squares Thermography," in 16th International Symposium on Applied Electromagnetics and Mechanics (ISEM16), Quebec City, 2013.

[8] R. Rosipal and N. Kramer, "Overview and recent advances in partial least," in Proceedings of Statistical and Optimization Perspectives, Bohinj, Slovenia, 2005.

[9] F. López and V. Nicolau, "Inverse heat transfer approach for IR image reconstruction: application to thermal non-destructive evaluation," Applied Thermal Engineering, vol. 33, 2012.

[10] F. López, V. Nicolau and X. Maldague, "Pulsed Phase Thermography applied on complex structures: modeling and numerical analysis," Naples, 2012.

[11] C. Ibarra-Castanedo, J. Piau, S. Guilbert, N. Avdelidis, M. Genest, A. Bendada and X. Maldague, "Comparative study of active thermography techniques for the nondestructive evaluation of honeycomb structures," Research in Nondestructive Evaluation, vol. 21, 2009.

[12] L. Mujica, J. Vehi, M. Ruiz, M. Verleysen, W. Staszewski and K. Worden, "Multivariate statistics process control for dimensionality reduction in structural assessment," Mechanical Systems and Signal Processing, vol. 22, 2008.

[13] H. Martens and T. Naes, Multivariate Calibration, Chichester, UK: John Wiley \& Sons, 1989.

[14] Eigenvector, Chemometrics Tutorial for PLS Toolbox and Solo, Wenatchee, WA: Eigenvector Research Inc., 2006.

[15] C. Ibarra-Castanedo, S. Sfarra, D. Ambrosini, D. Paoletti, A. Bendada and X. Maldague, "Subsurface defect characteriation in artworks by quantitative pulsed phase thermography and holographic interferometry," QIRT J., vol. 5, 2008. 